\title{
Alpha Cluster states in light nuclei populated through the $\left({ }^{6} \mathrm{Li}, \mathrm{d}\right)$ reaction
}

\author{
T.Borello-Lewin 1,a , M.R.D.Rodrigues ${ }^{a}$, H.Miyake ${ }^{a}$, L.B.Horodynski-Matsushigue ${ }^{a}$, \\ J.L.M.Duarte ${ }^{a}$, C.L.Rodrigues ${ }^{a}$, M.A.Souza ${ }^{a}$, A.Cunsolo ${ }^{b}$ F.Cappuzzello $^{b}$ and \\ G.M.Ukita ${ }^{\mathrm{C}}$ \\ ${ }^{a}$ Instituto de Física, Universidade de São Paulo \\ Caixa Postal 66318, CEP 05389-970, São Paulo, SP, Brazil \\ ${ }^{b}$ INFN - Laboratori Nazionali del Sud \\ via S.Sofia 62, 95125 Catania, Italy \\ ${ }^{c}$ Faculdade de Psicologia, Universidade de Santo Amaro \\ Rua Professor Eneas da Siqueira Neto, 340, CEP 04829-300, São Paulo, SP, Brazil \\ E-mail: borello@if.usp.br, marciadredfn.if.usp.br, lighia@if.usp.br, \\ jlduarte@if.usp.br, cleberlregmail.com, marsouzalif.usp.br, \\ miyakedif.usp.b, cunsolo@lns.infn.it, cappuzzello@lns.infn.it, \\ gmukita@uol.com.br
}

The ${ }^{12} \mathrm{C}\left({ }^{6} \mathrm{Li}, \mathrm{d}\right){ }^{16} \mathrm{O}$ reaction was used to investigate alpha resonant states in ${ }^{16} \mathrm{O}$ up to $17 \mathrm{MeV}$ of excitation. The reaction was measured at a bombarding energy of $25.5 \mathrm{MeV}$ employing the São Paulo Pelletron-Enge-Spectrograph facility and the nuclear emulsion detection technique. Several narrow resonances were detected and the resolution of $30 \mathrm{keV}$ allowed for the separation of doublets not resolved before.

XXXIV edition of the Brazilian Workshop on Nuclear Physics,

Foz de Iguaçu, Parana state, Brasil

5-10 June 2011

Speaker 


\section{Introduction}

The alpha cluster correlation is an important concept in the nuclear physics of light nuclei [1-4]. The main purpose of the research program in progress is the investigation of the alpha clustering phenomenon in $(\mathrm{x} \alpha)$ and $(\mathrm{x} \alpha+\mathrm{n})$ nuclei through the $\left({ }^{6} \mathrm{Li}, \mathrm{d}\right)$ alpha transfer reaction. In fact, there is scarce experimental information on the subject, in particular associated with oddeven nuclei and with resonant states predicted near $(x \alpha)$ and $(x \alpha+n)$ breakup thresholds $[5,6]$.

Focusing on alpha resonant states in the nucleus ${ }^{16} \mathrm{O}$, one state of special interest corresponds to the known $0^{+}$state at $15.1 \mathrm{MeV}$ of excitation that has probably the gas-like configuration of the $4 \alpha$ condensate state, with a very dilute density and a large component of $\alpha$ $+{ }^{12} \mathrm{C}($ Hoyle $)$ configuration [7,8]. On the other hand, the existence of a rotational band with the $\alpha+{ }^{12} \mathrm{C}$ (Hoyle) cluster state structure was recently demonstrated by Ohkubo and Hirabayashi [9]. In order to explore this region of interest, measurements of the ${ }^{12} \mathrm{C}\left({ }^{6} \mathrm{Li}, \mathrm{d}\right)$ reaction, at an incident energy of $25.5 \mathrm{MeV}$, have been performed employing the São Paulo Pelletron-Enge Split-Pole facility and the nuclear emulsion detection technique.

\section{Experimental Procedure}

A $25.5 \mathrm{MeV}{ }^{6} \mathrm{Li}$ beam of the São Paulo Pelletron Accelerator was focused on a clean and uniform ${ }^{12} \mathrm{C}$ target. The deuterons emerging from $\left({ }^{6} \mathrm{Li}, \mathrm{d}\right)$ reaction were momentum analyzed by the magnetic field of the Enge-Spectrograph and detected in emulsion plates (Fuji G6B, $50 \mu \mathrm{m}$ thick). Spectra associated with three scattering angles, $5^{\circ}, 8^{\circ}$ and $11^{\circ}$, in the laboratory frame, each one along $50 \mathrm{~cm}$ of the focal surface, were measured up to $17 \mathrm{MeV}$ excitation energy. After processing, the plates were scanned in strips of $200 \mu \mathrm{m}$ and the spectra were obtained, displaying the number of tracks per strip versus the position along the focal plane. An excellent energy resolution of $30 \mathrm{keV}$ was achieved mainly due to the careful determination of the focal plane of the reaction, the use of nuclear emulsion, uniform target, adequate spectrograph object and the good accelerator characteristics.

The relative normalization of the spectra and the absolute scale of the cross sections were, respectively, referred to the beam total charge collected in each run and to optical model predictions for elastic scattering measurements in the same target and under similar conditions.

\section{Comments and results}

The ${ }^{12} \mathrm{C}\left({ }^{6} \mathrm{Li}, \mathrm{d}\right)$ reaction was measured by the first time with a resolution of $30 \mathrm{keV}$ allowing the discrimination of several doublets . Fig. 1 shows the position deuteron spectrum associated with the scattering angle of $5^{0}$. The $\mathrm{J}^{\pi}$ and excitation energies in $\mathrm{MeV}$ of the detected states taken from Tilley et al. [10] are indicated. Several narrow resonances were detected in the range of 10-17 MeV excitation energy, with a quasi-bound behavior, embedded in the continuum. In particular, three doublets that were not resolved previously are around the $4 \alpha$ threshold at $14.4 \mathrm{MeV}$. These doublets suggest a possible band structure. 


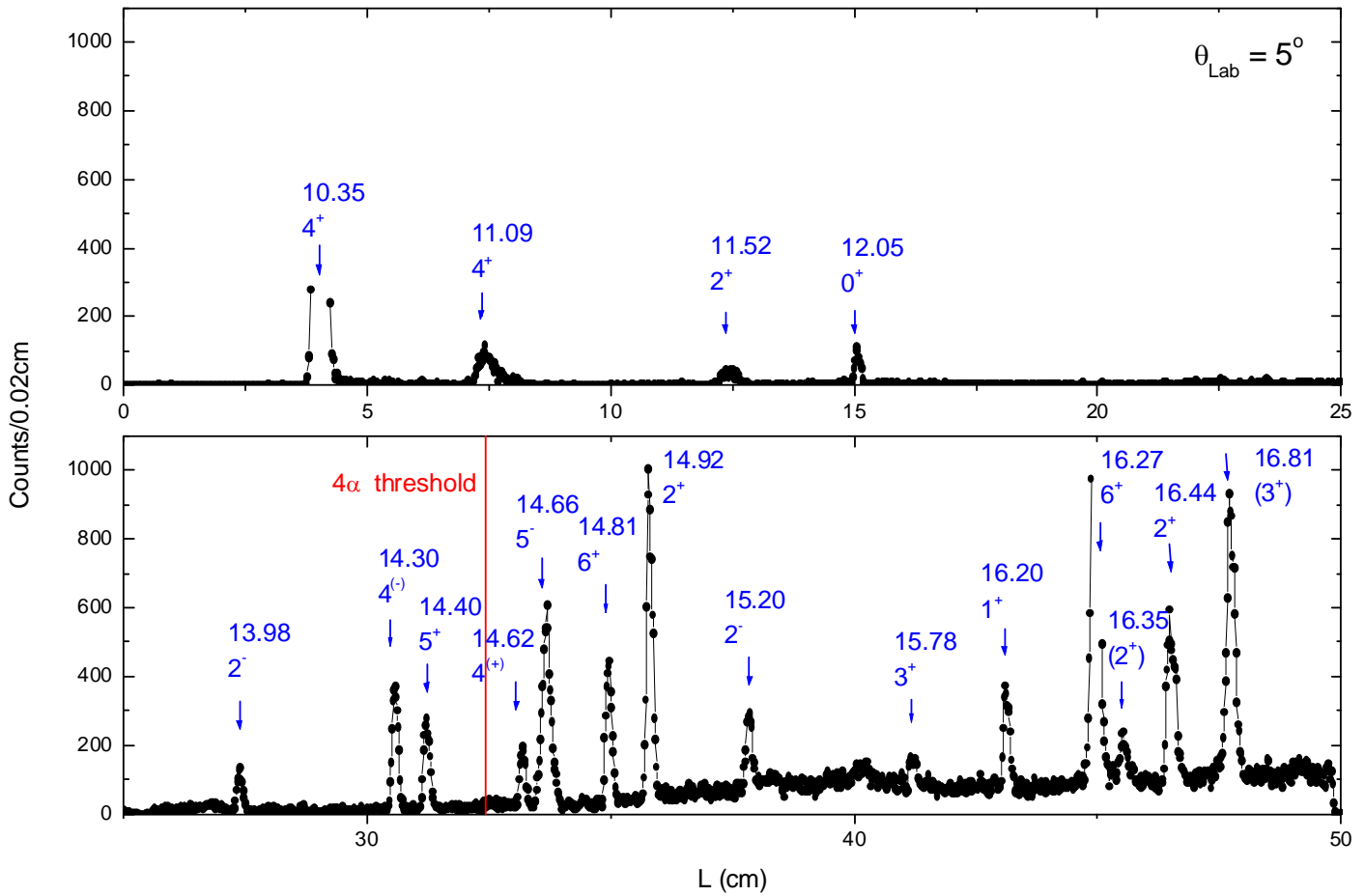

Fig1: Position deuteron spectrum. The $\mathrm{J}^{\pi}$ and excitation energies in $\mathrm{MeV}$ associated with each state from Ref. [10] are indicated. The $4 \alpha$ threshold is also shown. The peaks at 10.35 and 16.27 $\mathrm{MeV}$ appear super exposed in this spectrum.

Note that the doublet resolved in the present work just above the $4 \alpha$ threshold, associated with $\mathrm{J}^{\pi}=4^{(+)}$and $5^{-}$at 14.62 and $14.66 \mathrm{MeV}$ excitation energies, respectively [10], was also observed by Ames [11] in alpha elastic scattering on ${ }^{12} \mathrm{C}$ excitation function. On the other hand, Wheldon et al. [12] detected through ${ }^{12} \mathrm{C}\left({ }^{6} \mathrm{Li}, \mathrm{d}\right)$ reaction at a higher incident energy, only one state at $14.6 \mathrm{MeV}$ associated with a strongly populated broad resonance which decays exclusively to the ${ }^{12} \mathrm{C}$ ground state.

In the region of the $0^{+}$state at $15.1 \mathrm{MeV}$ of excitation that has probably the gas-like configuration of the $4 \alpha$ condensate state, was detected only one state, most like the state $\mathrm{J}^{\pi}=2^{-}$ at $15.20 \mathrm{MeV}$, as also indicated by Wheldon et al.[12]. However, only a detailed analysis of the angular distribution will clarify completely this question.

The absolute cross sections for the resonances at the three scattering angles measured are in the range of $10^{-2}-10^{-1} \mathrm{fm}^{2} / \mathrm{sr}$. Complementary acquisition data is already scheduled, in order to have a full angular distribution. It is important to note that the present work is in progress and further analysis will contribute with new information in this region of renewed interest [13]. 


\section{Conclusion}

Several narrow resonances were detected in the range of 10-17 MeV of excitation energy. The discrimination of several doublets, allowed by the good resolution of the data, suggests a band structure in this region. The present work is in progress, with complementary measurement scheduled and further analysis undergoing. The preliminary results presented here indicate that the good resolution achieved in addition with a detailed angular distribution to be measured will resolve some parities doubts and give new information in this region of interest.

\section{References}

[1] K. Ikeda, N. Takigawa and H. Horiuchi, Prog. Theor. Phys. Suppl. 464-475 (1968).

[2] Y. Fujiwara, H. Horiuchi, K. Ikeda, M. Kamimura, K. Katō, Y. Suzuki and E. Uegaki, Prog. Theor. Phys. Sup. 68, 29 (1980) and references therein.

[3] P. Descouvemont, Phys. Rev. C 44, 306 (1991)

[4] S. Ohkubo et al., Prog. Theor. Phys. Sup. 132, 1 (1998) and references therein.

[5] M. R. D. Rodrigues et al., 12th International Conference on Nuclear Reaction Mechanism, Varenna, Italy 2009, edited by F. Cerutti and A. Ferrari, CERN Proc. 01, 331-335 (2010).

[6] T. Borello-Lewin et al., Proceedings of SOTANCP2, Brussels, Belgium 2010, edited by P. Descouvemont et al., Int. J. Mod. Phys E 20, 1018-1021(2011).

[7] Y. Funaki, T. Yamada, H. Horiuchi, G. Ropke, P. Schuck,A. Tohsaki, Phys. Rev. Lett. 101, 082502 (2008)

[8] Y. Funaki, T. Yamada, A. Tohsaki, H. Horiuchi, G Ropke, and P. Schuck, Phys. Rev. C 82, 024312 (2010)

[9] S. Okubo and Y. Hirabayashi, Phys. Lett. B 681, 127-131 (2010).

[10] D. R. Tilley et al., Nucl. Phys. A 565, 1-184 (1993)

[11] L. L. Ames et al., Phys. Rev. C 25, 729 (1982)

[12] C. Wheldon et al., Phys. Rev. C 83, 064324 (2011)

[13] “Clusters in Nuclei”, Lect. Notes Phys. 818, ed. C. Beck, Springer Heidelberg (2010). 